\title{
Propagation Characteristics in a Planar Dielectric Waveguide with Left-handed Medium
}

\author{
Pu Jiapeng, Lu Guizhen \\ Information School, Communication University of China, BeiJing, China \\ pujiapeng@cuc.edu.cn, luguizhen@cuc.edu.cn
}

\begin{abstract}
- this paper has studied electromagnetic waves in planar dielectric waveguide containing left-handed medium propagate characteristics, the mode of transmission with left-hand materials is analyzed theoretically. Through the analysis of TE and TM wave in the transmission characteristics of planar dielectric waveguide, TE and TM mode dispersion equation are obtained, Concluded that the electromagnetic wave in the spread of the planar dielectric waveguide containing left-handed medium not only had oscillation wave and surface wave, traditional media planar dielectric waveguide only have the shock wave. Secondly, Simulation analysis has also been made on the propagation characteristics in slab waveguide with left-hand materials, Compared to traditional media planar dielectric waveguide, Planar dielectric waveguide containing left-handed medium compared with traditional planar dielectric waveguide possesses the advantages of larger bandwidth and less attenuation, then proved that this structure can be used as the transmission lines of microwave circuit.
\end{abstract}

Index Terms - left-handed medium, transmission characteristics

COMSOL

\section{Introduction}

Permittivity $\varepsilon$ and magnetic permeability $\mu$ are two basic physical quantities to describe the electromagnetic properties. For the conventional positive refractive materials, both of the permittivity and permeability are greater than zero, and the Maxwell equations has proved that electromagnetic waves will form the right-handed helical relationships when it propagates electric field, magnetic field and the wave vector in the positive refractive material, thus it is also called as the righthand materials.

In 1968, Soviet scientists V.G. Veselago analyzed the electromagnetic properties of isotropic homogeneous medium theoretically, when both of permittivity and magnetic permeability are negative simultaneously, such as: the inverse Snell effect, the inverse Doppler effect, the inverse Qilun Markov radiation, negative light pressure phenomena and so on[1]. When electromagnetic waves propagates in such medium, its electric, magnetic and wave vector will form the three left-handed helical relationship, so Veselago named such medium as left-handed media. In recent years, with the negative refractive index materials of microwave synthetic has become the truth[2], the study of negative refractive index materials has become the focus in international academic physics and electromagnetism[3-7].In 2002, G.V.Eleftheriades in University of Toronto with his team used transmission line theory proved that in two-dimensional transmission line network load shunt inductor $\mathrm{L}$ and capacitor $\mathrm{C}$ in series consisting of a high -pass LC transmission line network under certain conditions have a negative $\mathrm{e}^{[8-9]}$ permittivity and negative permeability by such network elements constitute a twodimensional left-handed dielectric waveguide This paper mainly studies the propagation characteristics in planar waveguide with left-hand materials in the case of electromagnetic waves in two-dimensional, the mode of transmission with left-hand materials is analyzed theoretically, Simulation analysis has also been made on the propagation characteristics in planar waveguide with left-hand materials. Compared to traditional media planar dielectric waveguide, the planar waveguide with left-hand materials has better propagation properties.

\section{The Basic Principle of Left-Handed Medium}

Electromagnetic wave propagation in the medium behavior is determined by the dielectric constant $\varepsilon$ and magnetic permeability $\mu$.A beam of plane wave spread in isotropic homogeneous medium, The wave vector $\vec{k}$ and frequency $w$ satisfy the dispersion relation

$$
k^{2}=\frac{w^{2}}{c^{2}} n^{2}\left(n^{2}=\varepsilon \mu, c^{2}=\frac{1}{\varepsilon_{0} \mu_{0}}\right)
$$

Among, refractive index is $n, c$ is the speed of light in a vacuum. If you don't consider any energy loss, in a normal medium $n, \varepsilon, \mu$ are all positive number. $\varepsilon$ and $\mu$ are negative real number at the same time, On the surface, The dispersion relations is not affected by any. But fundamentally, It is derived from Maxwell's equations of electromagnetic field. Monochromatic plane wave propagation in isotropic passive medium to meet the equation for the Maxwell's equations and the media's equations

$$
\begin{gathered}
\nabla \times \vec{E}=-\frac{\partial B}{\partial t}, \nabla \times \vec{H}=-\frac{\partial D}{\partial t} \\
\vec{E}=\overrightarrow{E_{0}} e^{i(\vec{k} \cdot \vec{r}-w t)} \vec{H}=\overrightarrow{H_{0}} e^{i(\vec{k} \cdot \vec{r}-w t)} \text { in the above two type }
\end{gathered}
$$

available

$$
\vec{k} \times \vec{E}=\frac{w}{c} \cdot \mu \vec{H}, \vec{k} \times \vec{H}=\frac{w}{c} \cdot \varepsilon \vec{H}
$$

Thus it can be seen, When $\varepsilon$ and $\mu$ at the same time is greater than zero, Electromagnetic wave vector $\vec{k}$, the electric 
vector $\vec{E}$ and magnetic vector $\vec{H}$ constitute a right relationship. And when $\varepsilon$ and $\mu$ at the same time is less than zero, they constitute a right relationship, and the wave vector $\vec{k}$ and the poynting vector $\vec{S}=\vec{E} \times \vec{H}$ in the opposite direction, The phase velocity and group velocity in the opposite direction. The physical nature of negative permittivity $\varepsilon$ and permeability $\mu$ can be explained by Drude - Lorentz model. Assume the atoms and molecules in the material can be thought of in a electronics the bondage of inherent frequency $w_{0}$ resonant harmonic oscillator. Under the action of electric field, When the frequency of the electric field $w<<w_{0}$, A displacement relative to the nucleus, and the outer one induced polarization electric field direction, the polarization direction and an electric field at the same direction, the dielectric constant is positive. At the same time $w \rightarrow w_{0}$, resonant harmonic oscillator with electric field, field induced polarization is very big, there is a lot of energy in the harmonic oscillator. When the electric field direction reversals, the polarization direction of the harmonic oscillator is almost unaffected. When frequency close to the resonance frequency $w_{0}$,Polarization of harmonic oscillator By the phase shift with electric field for an electric field and the reverse phase, Thus the negative effects.

\section{Theoretical Foundation}

Slab waveguide with left-hand materials is shown in Figure 1:

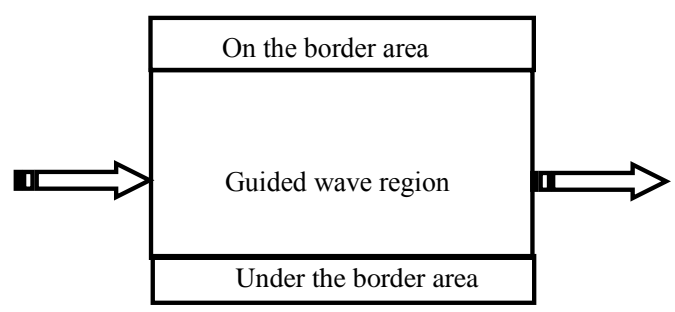

Figure1 Slab waveguide with left-hand materials

The top and bottom of slab waveguide are the same, both of which are interface region with left-hand materials, relative dielectric constant $\varepsilon_{1}$, relative permeability $\mu_{1}$, and the refractive index $n_{1}=-\sqrt{\varepsilon_{1} \mu_{1}}$ are less than zero; Guided wave region is air, the thickness of guided wave region is $2 d$, relative dielectric constant and relative permeability were $\varepsilon_{2}$ and $\mu_{2}$, Refractive index $n_{2}$, and $\varepsilon_{2}=1, \mu_{2}=1, n_{2}=1$.

Using the classical Maxwell equations, combined with continuity boundary conditions, the dispersion equation of TE mode can be conculed;

$$
p d=\frac{\mu_{1}}{\mu_{2}} k d \tan \left(k d-\frac{m \pi}{2}\right)
$$

Wherein, while the attenuation coefficient of the interfacial region $p=\sqrt{\beta^{2}-k_{0}^{2} n_{1}^{2}}$, the propagation coefficient of guided wave region $k=\sqrt{k_{0}^{2} n_{2}^{2}-\beta^{2}}, \beta$ is the propagation constant, $k_{0}$ is the vacuum wave vector. In fact, the dispersion equation for conventional transmission media also established. In this study, since $n_{1}<0$, the characteristics will be given by the equation is different from slab waveguide with conventional materials .The following can be obtained from above ;

$$
(p d)^{2}+(k d)^{2}=k_{0}^{2} d^{2}\left(n_{2}^{2}-n_{1}^{2}\right)=a^{2}
$$

Where a is defined as the normalized frequency (or normalized thickness). conventional materials of planar dielectric waveguide,requiring $\varepsilon_{2} \mu_{2}>\varepsilon_{1} \mu_{1}$,That the refractive index $n_{2}>n_{1}>0, N=\beta / k_{0}$ is the refractive index in the range $n_{1}<N<n_{2}$. And because the phase velocity $V_{p}=w / \beta>c / n_{2}$, so often referred to as the fast wave. Formula (4), $\mathrm{m}$ is an integer value of $0,1,2 \cdots .$. , corresponding to a different order of guided wave $T E_{0}, T E_{1}$, $T E_{2} \cdots \cdots$, But for a planar dielectric waveguide with lefthanded medium, $\mathrm{K}$ can be the imaginary number even when $\varepsilon_{2} \mu_{2}>\varepsilon_{1} \mu_{1}$, which means that not only oscillation wave but also evanescent wave exist in the guided wave region, and it is actually a surface wave propagation at the interface. So, a planar dielectric waveguide with left-handed medium has better propagation properties. At this time, the phase velocity $V_{p}<c / n_{2}$, this wave is a slow wave, surface waves cannot be propagated by conventional planar dielectric. For the TM mode, using electromagnetic duality relation can get a similar dispersion equation easily;

$$
p d=\frac{\varepsilon_{1}}{\varepsilon_{2}} k d \tan \left(k d-\frac{m \pi}{2}\right)
$$

\section{Planar Dielectric Waveguide with Left-handed Medium}

According to the upper and lower planar dielectric waveg uide boundary region and the refractive index of the waveguid e region of the positive and negative pole, Planar dielectric waveguide with left-handed medium into three forms, namely, "negative - positive - negative","positive - negative - positive" and "negative-negative-negative", Specific data such as tableIIV. According to the use of COMSOL software simulation in three forms of TE wave propagation characteristics and with the conventional planar dielectric waveguides "positivepositive-positive" for comparison, it is found that planar 
dielectric waveguide with left-handed medium is better propagation properties.

Table I the data of "positive -positive-positive"

\begin{tabular}{|c|c|c|c|c|}
\hline & $\begin{array}{c}\text { Dielectric } \\
\text { constant } \varepsilon\end{array}$ & $\begin{array}{c}\text { Magnetic } \\
\text { permeability } \mu\end{array}$ & $\begin{array}{c}\text { Length } \\
(\mathrm{m})\end{array}$ & $\begin{array}{c}\text { Width } \\
(\mathrm{m})\end{array}$ \\
\hline On the border area & 2 & 2 & 0.1 & 0.01 \\
\hline Guided wave area & 5 & 5 & 0.1 & 0.1 \\
\hline Under the border area & 2 & 2 & 0.1 & 0.01 \\
\hline
\end{tabular}

Table II the data of "negative - positive - negative"

\begin{tabular}{|c|c|c|c|c|}
\hline & $\begin{array}{c}\text { Dielectric } \\
\text { constant } \varepsilon\end{array}$ & $\begin{array}{c}\text { Magnetic } \\
\text { permeability } \mu\end{array}$ & $\begin{array}{c}\text { Length } \\
(\mathrm{m})\end{array}$ & $\begin{array}{c}\text { Width } \\
(\mathrm{m})\end{array}$ \\
\hline On the border area & -2 & -2 & 0.1 & 0.01 \\
\hline Guided wave area & 5 & 5 & 0.1 & 0.1 \\
\hline Under the border area & -2 & -2 & 0.1 & 0.01 \\
\hline
\end{tabular}

Table III the data of "positive - negative - positive"

\begin{tabular}{|c|c|c|c|c|}
\hline & $\begin{array}{c}\text { Dielectric } \\
\text { constant } \varepsilon\end{array}$ & $\begin{array}{c}\text { Magnetic } \\
\text { permeability } \mu\end{array}$ & $\begin{array}{c}\text { Length } \\
(\mathrm{m})\end{array}$ & $\begin{array}{c}\text { Width } \\
(\mathrm{m})\end{array}$ \\
\hline On the border area & 2 & 2 & 0.1 & 0.01 \\
\hline Guided wave area & -5 & -5 & 0.1 & 0.1 \\
\hline Under the border area & 2 & 2 & 0.1 & 0.01 \\
\hline
\end{tabular}

Table IV the data of "negative -negative-negative"

\begin{tabular}{|c|c|c|c|c|}
\hline & $\begin{array}{c}\text { Dielectric } \\
\text { constant } \varepsilon\end{array}$ & $\begin{array}{c}\text { Magnetic } \\
\text { permeability } \mu\end{array}$ & $\begin{array}{c}\text { Length } \\
(\mathrm{m})\end{array}$ & $\begin{array}{c}\text { Width } \\
(\mathrm{m})\end{array}$ \\
\hline On the border area & -2 & -2 & 0.1 & 0.01 \\
\hline Guided wave area & -5 & -5 & 0.1 & 0.1 \\
\hline Under the border area & -2 & -2 & 0.1 & 0.01 \\
\hline
\end{tabular}

When propagates in planar dielectric waveguide, electromagnetic wave is periodical, and the narrower the upper and lower boundary region are, the broader the band is; the smaller the refractive index is, the broader the band is. Providing that the upper and lower boundary region and refractive index are certain, one may examine the positive or negative of refractive index on the propagation properties. Shaped like a "positive - positive - positive" in the form of band in Figure 2. Shaped like "negative - positive - negative", "positive - negative - positive" and "negative -negativenegative" in the form of band in Figure 3.

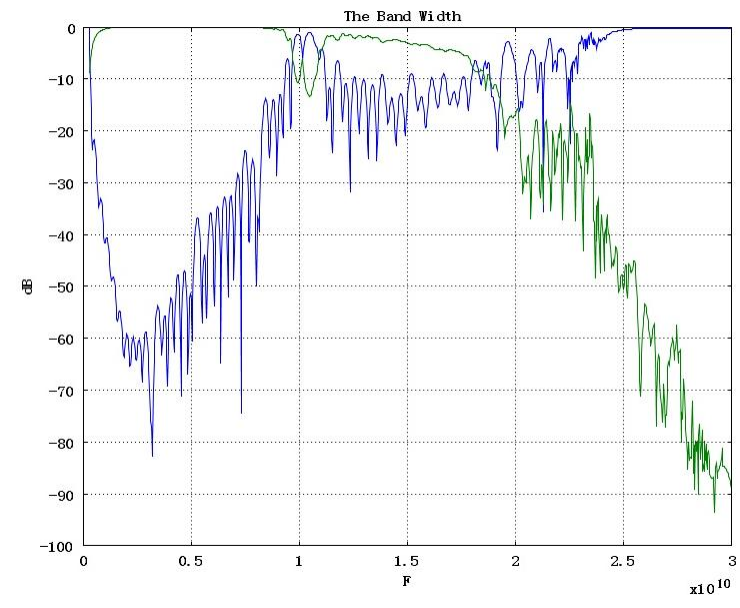

Figure 2 Conventional planar waveguide bandwidth

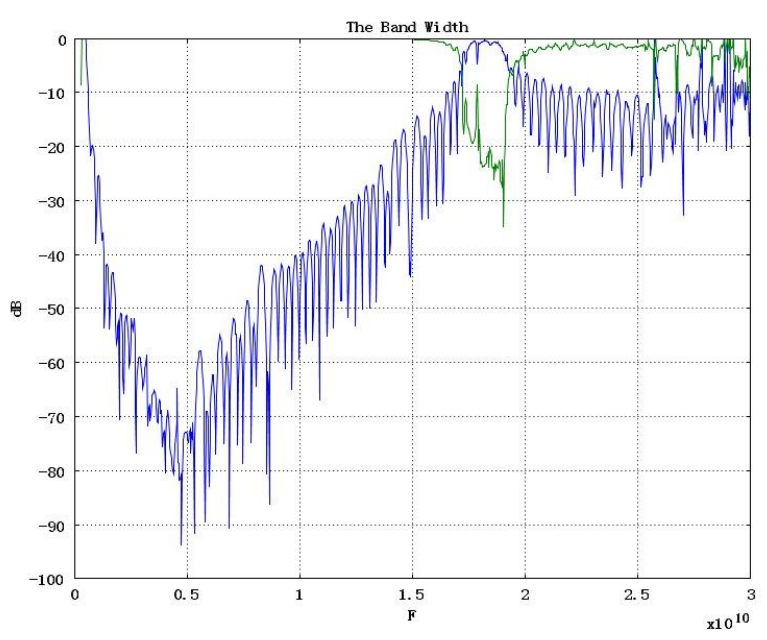

Figure 3 Planar dielectric waveguide with left-handed medium

Contrasted Figure 2 to Figure 3, when the refractive index and the boundary region is the same, planar dielectric waveguide with left-handed media has a large bandwidth and less attenuation compared to conventional planar dielectric waveguide.

\section{Conclusions}

This paper analyzed theoretically electromagnetic waves in the planar dielectric waveguide with left-handed medium not only oscillation wave but also evanescent wave exist in the guided wave region, and it is actually a surface wave propagation at the interface. But electromagnetic waves in the conventional planar dielectric waveguide only have oscillation wave, not have surface wave. Through COMSOL software simulation experiments proved that the planar dielectric waveguide with left-handed medium compared with traditional media planar dielectric waveguide possesses the advantages of larger bandwidth and less attenuation, That planar dielectric waveguide with left-handed medium compared to traditional planar dielectric waveguide has better performance of guided wave. 


\section{References}

[1] V.G.Veselago. The electrodynamics of substances with simultaneously negative values of $\varepsilon$ and $\mu$. Soviet Physics Uspekhi, 1968, 10(4): 509514.

[2] D.R.Smith, W.J.Padilla, D.C.Vier, et al.Composite medium with Simultaneously negative permeability and permittivity. Physical Review Letter, 2000, 84(18):4184-4187.

[3] Shelby R A,Smith D R,Schultz S.Experimental verification of a negative index of refraction. Science, 2001, 292:77-79.

[4] Greegor R, Parazzoli C, Li K, et al.Experimental determination and Numerical simulation of the Properties of negative index of refraction materials. Optics Express, 2003, 11(7):688-695.
[5] Marques R, Baena J.Effect of losses and dispersion on the focusing properties of Left-Handed media. Micro-wave and Optical Technology Letters, 2004, 41(4): 290-294

[6] Qing D, Chen Gang. Goos-Haenchen shifts at the inter-faces between left- and right-handed media. Optics Letters, 2004, 8:872-874.

[7] Wu B,Grzegorczyk T M, Zhang Y, et al.Guided modes with imaginary transverse wave number in a slab waveguide with negative permittivityand permeability. Journal of Applied Physics, 2004, 93(11): 9386-9388.

[8] G. V .Eleftheriades, A.K.Iyer, P.C.Kremer. Planar negative refractive index media Using periodically L-C loaded transmission lines. IEEE on Transactions Microwave Theory and Techniques, 2002, 50(12): 27022712. 